Bull. Mater. Sci., Vol. 6, No. 2, May 1984, pp. 263-272. (C) Printed in India.

\title{
Structural and magnetic investigations of some new boron containing rare earth intermetallic compounds
}

\author{
S K MALIK, S K DHAR and R VIJAYARAGHAVAN \\ Tata Institute of Fundamental Research, Bombay 400005 , India
}

\begin{abstract}
By alloying boron with $\mathbf{R P d}_{3}(\mathbf{R}=$ rare earth) compounds, new ternary alloys of the formula $\operatorname{RPd}_{3} \mathrm{~B}_{x}(0 \leqslant x \leqslant 1)$ have been prepared. The parent $\mathbf{R P d}_{3}$ compounds crystallise in the cubic $\mathrm{AuCu}_{3}$ type structure. The addition of boron does not change the structure but results in an expansion of the lattice. Therefore, it is likely that the small boron atoms occupy the vacant body centred position in $\mathrm{AuCu}_{3}$ type structure. It is also observed that compounds of the composition $R R_{h_{3}} B$ can be formed in the cubic structure for all rare earths $R$ though the parent $R R_{3}$ compounds exist only for $\mathrm{R}=\mathrm{Ce}\left(\mathrm{AuCu}_{3}\right.$ type) and $\mathrm{La}, \mathrm{Sm}, \mathrm{Nd}$ and $\mathrm{Gd}$ (all hexagonal $\mathrm{CeNi}_{3}$ type). This points out the role of boron in stabilising new crystallographic phases. The results of susceptibility measurements on some of the $\mathbf{R P d}_{3} \mathbf{B}$ and $\mathbf{R R h}_{\mathbf{3}} \mathbf{B}$ compounds are presented. In particular, it is noted that while $\mathrm{GdPd}_{3}$ orders antiferromagnetically with $T_{N}=6 \mathrm{~K}, \mathrm{GdPd}_{3} \mathrm{~B}$ does not order magnetically down to $4 \cdot 2 \mathrm{~K}$.
\end{abstract}

Keywords. Boron: rare earths; magnetic susceptibility; structural investigations.

\section{Introduction}

During the last few years a new class of rare earth compounds known as rare earth ternary borides have attracted a great deal of attention. The interest in these compounds is due to their fascinating magnetic and superconducting properties. For example, in the series of ternary borides represented by the formula $R R_{4} B_{4}(R=$ rare earth), first synthesized by Matthias et al $(1977 \mathrm{a}, \mathrm{b})$, the compounds with Th, Y, Nd, Sm, $\mathrm{Er}, \mathrm{Tm}$ and $\mathrm{Lu}$ are superconducting whereas those with $\mathrm{Gd}, \mathrm{Tb}, \mathrm{Dy}$ and Ho undergo ferromagnetic ordering. Following the $\mathrm{RRh}_{4} \mathrm{~B}_{4}$ series of compounds, many other ternary borides forming at different stoichiometries have been synthesised and investigated for magnetic and possible superconducting behaviour. In this paper we report the formation of new boron containing rare earth compounds represented by the formula $\mathbf{R P d}_{3} \mathrm{~B}_{x}(0 \leqslant x \leqslant 1)$ and $R \mathrm{Rh}_{3} \mathrm{~B}$ where $\mathbf{R}=\mathrm{La}$ to Lu. Magnetic studies on some of these alloys are also presented.

An indication that alloys of the formula $\mathrm{RRh}_{3} \mathrm{~B}_{x}$ and $\mathrm{RPd}_{3} \mathrm{~B}_{x}$ may well exist came first during the course of our investigations on $R \mathrm{Rh}_{3} \mathrm{~B}_{2}$ compounds described elsewhere (Malik et al 1983). In the initial stages of our work on these, we observed, in the $x$-ray diffraction pattern of $\mathrm{CeRh}_{3} \mathrm{~B}_{2}$, the existence of some extra lines which could not be ascribed to hexagonal $C e \mathrm{R}_{3} \mathrm{~B}_{2}$ compound. A careful analysis showed that these extra lines could be indexed on the basis of a cubic structure. The lattice constant of this cubic phase turned out to be slightly larger than that of $\mathrm{CeRh}_{3}$ which crystallises in the cubic $\mathrm{AuCu}_{3}$ structure type (Harris and Norman 1967). Because of the structural considerations of the $\mathrm{AuCu}_{3}$ type crystal lattice, to be discussed below, the formula of this new cubic phase was presumed to be $\mathrm{CeRh}_{3} \mathrm{~B}_{x}(0<x \leqslant 1)$. Subsequently we found that starting with appropriate amounts of $\mathrm{Ce}, \mathrm{Rh}$ and $\mathrm{B}$, cubic compounds having the formula $\mathrm{CeRh}_{3} \mathrm{~B}_{x}(0<x \leqslant 1)$ could indeed be formed. The formation of alloys of the 
above composition immediately suggested the likely existence of $\operatorname{RPd}_{3} \mathrm{~B}_{x}$ compounds. The $\mathbf{R P d}_{3}$ compounds also crystallise in the $\mathrm{AuCu}_{3}$ structure type (Harris and Raynor 1965) with lattice constants comparable to that of $\mathrm{CeRh}_{3}$. Later investigations (Dhar et al 1981a) verified the formation of $\operatorname{RPd}_{3} B_{x}(0<x \leqslant 1)$ alloys for all the rare earths $R$. Further extension of the work showed that $\mathrm{RPd}_{3} \mathrm{Si}_{x}$ alloys could also be formed, though silicon content is much smaller.

\section{2. $\operatorname{RPd}_{3} \mathbf{B}_{x}(0<x \leqslant 1 ; R=$ Rare earth $)$ alloys}

\subsection{Synthesis and structure}

Master $\mathbf{R P d}_{3}$ alloys were first prepared by melting together stoichiometric amounts of the rare earth and the Pd metal in an arc furnace. The alloy ingots were turned and melted several times to ensure homogeneity. Approximately half the amount of each $\mathbf{R P d}_{3}$ alloy was remelted with an appropriate amount of boron in a water cooled copper boat in an induction furnace to form $\mathrm{RPd}_{3} \mathrm{~B}$ alloys. This preliminary mixing of boron with $\operatorname{RPd}_{3}$ achieved by induction heating was necessary as otherwise boron chunks were found to shatter and fly away on coming under the arc in the arc furnace. In order to ensure complete mixing the fused charge of $\mathrm{RPd}_{3}$ and boron was remelted in the arc furnace. Alloy formation at the stoichiometry $\mathrm{RPd}_{3} \mathrm{~B}_{x}(0<x \leqslant 1)$ was tried for few rare earths only, e.g. $\mathrm{La}, \mathrm{Ce}, \mathrm{Pr}, \mathrm{Eu}, \mathrm{Gd}$ and $\mathrm{Dy}$ and these were obtained by remelting appropriate proportions of $\mathrm{RPd}_{3}$ and $\mathrm{RPd}_{3} \mathrm{~B}$. In the case of europium and ytterbium compounds, excess amount of the rare earth was taken to compensate for the loss due to evaporation during melting. Powder $x$-ray diffraction patterns were recorded using $\mathrm{CuK}_{\alpha}$ radiation.

The $\mathrm{RPd}_{3}$ compounds are known to crystallise in the cubic $\mathrm{AuCu}_{3}$ type structure, space group $\mathrm{Pm} 3 m$ (figure la). The rare earth atoms occupy the cube corners and Pd atoms are on the face centred positions. The various atomic positions in the unit cell are given by (Pearson 1967)
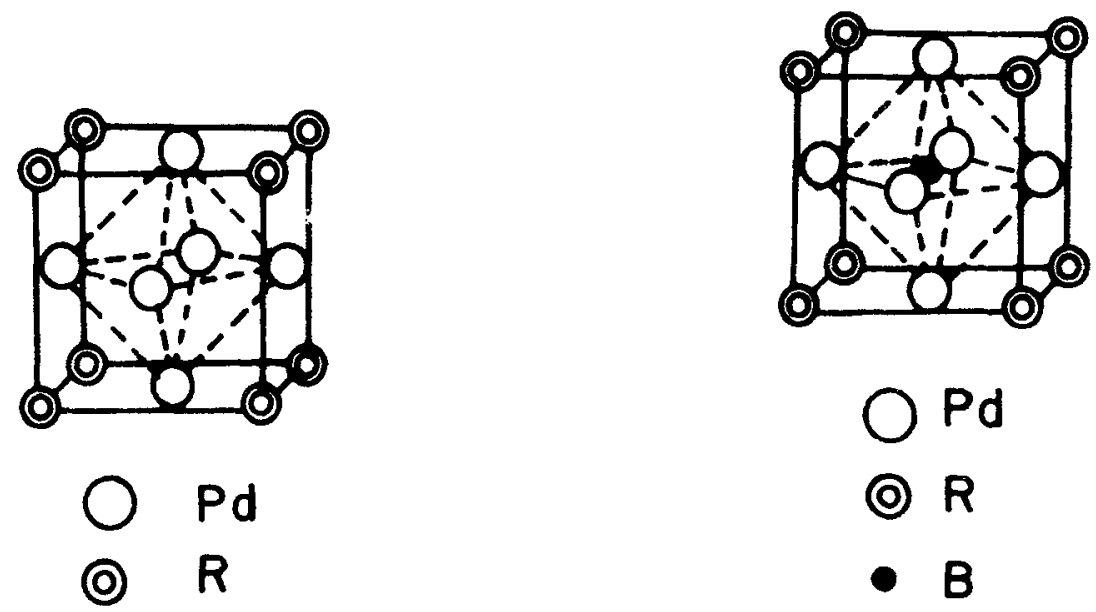

Figure 1a. The unit cell of $\mathbf{R P d}_{3}\left(\mathrm{R}=\right.$ rare earth) compounds crystallising in $\mathrm{AuCu}_{3}$ type structure. b. The proposed crystal structure of $\mathbf{R P d}_{3} \mathbf{B}$ compounds. 


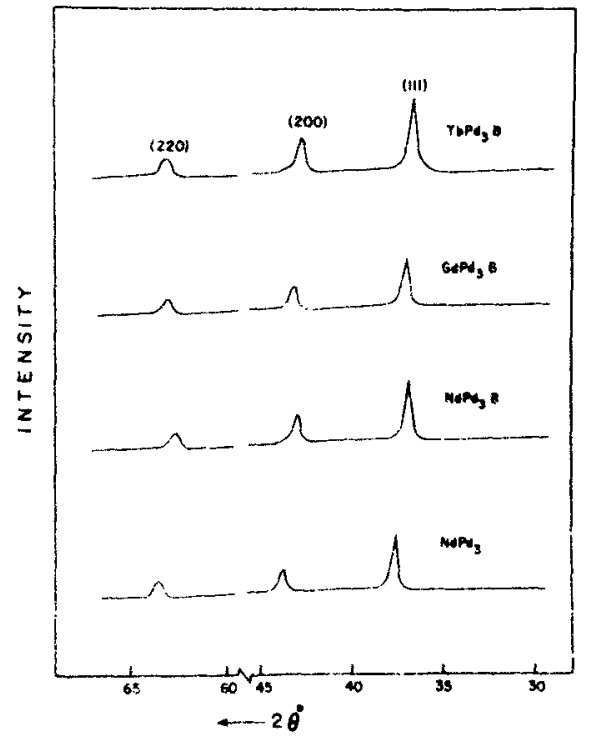

Figure 2. The $x$-ray diffraction patterns of $\mathrm{NdPd}_{3}$ and some $\mathrm{RPd}_{3} \mathrm{~B}(\mathrm{~K}=\mathrm{Nd}, \mathrm{Gd}$ and $\mathrm{Tb})$ compounds.

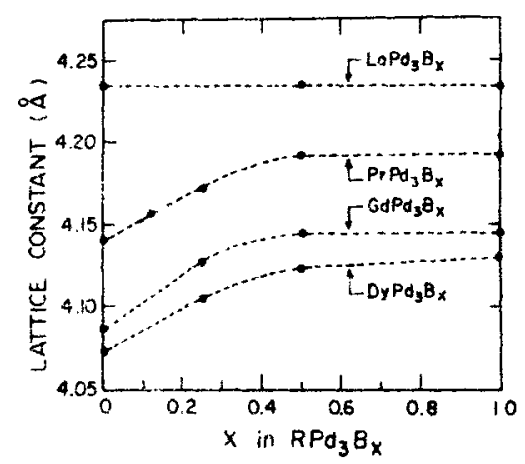

Figure 3. Lattice parameter of $\operatorname{RPd}_{3} B_{x}$ $(R=\mathrm{La}, \mathrm{Pr}, \mathrm{Gd}$ and Dy) alloys as a function of boron concentration $x$.

$$
\begin{array}{llll}
\mathrm{R} & (1 a) & \mathrm{m} 3 m & : 0,0,0 \\
\mathrm{Pd} & (3 c) & 4 / \mathrm{mmm} & : 0,1 / 2,1 / 2 ; 1 / 2,0,1 / 2 ; 1 / 2,1 / 2,0 .
\end{array}
$$

The $x$-ray diffraction patterns of $\mathrm{RPd}_{3} \mathrm{~B}_{x}$ (for all $x$ ) compounds are identical to that of the $\mathrm{RPd}_{3}$ compounds (figure 2) and hence can be fitted to cubic structure type. However, the lattice parameter of the former is observed to be slightly larger than that of the parent $\mathrm{RPd}_{3}$ compound and depends on the boron concentration $x$. Figure 3 shows the dependence of lattice parameter on boron concentration in some of these alloys. The relatively small boron atoms presumably occupy the vacant body-centred position in the $\mathrm{RPd}_{3}$ unit cell to give rise to an $E 2_{1}$ anti-perovskite arrangement, space group $\mathrm{Pm} 3 m$ (figure $1 \mathrm{~b}$ ). Thus $\mathrm{RPd}_{3} \mathrm{~B}_{x}$ alloys may be considered to result from "addition" of boron to parent $\mathrm{RPd}_{3}$ compound. Many compounds which have the $\mathrm{AuCu}_{3}$ type structure are known to accommodate smaller non-metallic elements like $\mathrm{B}$, $\mathrm{C}, \mathrm{N}, \mathrm{Si}$ etc. at the body-centred position resulting in anti-perovskite compound formation (Gschneidner et al 1978). The various atomic positions in the $\mathrm{RPd}_{3} \mathrm{~B}$ unit celi are given by (Pearson 1967)

$$
\begin{array}{llll}
\mathrm{R} & (1 a) & m 3 m & : 0,0,0 \\
\mathrm{Pd} & (3 c) & 4 / m m m: 0,1 / 2,1 / 2 ; 1 / 2,0,1 / 2 ; 1 / 2,1 / 2,0 . \\
\mathrm{B} & (1 b) & m 3 m & : 1 / 2,1 / 2,1 / 2 .
\end{array}
$$

Table 1 lists the lattice constants of $\mathrm{RPd}_{3}$ and $\mathrm{RPd}_{3} \mathrm{~B}$ compounds and the same information is plotted graphically in figure 4 . It should be noted that the expansion in the cell volume of $\mathrm{Ce}$ and $\mathrm{Eu}$ compounds is relatively larger. Because of the highly volatile nature of $\mathrm{Yb}$, efforts to make single phase $\mathrm{YbPd}_{3} \mathrm{~B}$ did not succeed. However, the extra phase in the alloy is estimated to be less than $10 \%$. 
Table 1. Lattice constants of $R \mathrm{Pd}_{3}$ and $\mathrm{RPd}_{3} \mathbf{B}(\mathbf{R}=$ rare earth) compounds

\begin{tabular}{|c|c|c|c|}
\hline Compound & $\begin{array}{c}\text { Lattice } \\
\text { constant } \\
\text { a }(\AA)\end{array}$ & Compound & $\begin{array}{c}\text { Lattice } \\
\text { constant } \\
a(\AA)\end{array}$ \\
\hline $\mathrm{LaPd}_{3}$ & $4 \cdot 229$ & $\operatorname{LaPd}_{3} B$ & $4 \cdot 229$ \\
\hline $\mathrm{CePd}_{3}$ & $4 \cdot 124$ & $\mathrm{CePd}_{3} \mathrm{~B}$ & $4 \cdot 203$ \\
\hline $\mathbf{P r P d}_{3}$ & $4 \cdot 141$ & $\operatorname{PrPd}_{3} B$ & $4 \cdot 194$ \\
\hline $\mathrm{NdPd}_{3}$ & $4 \cdot 132$ & $\mathrm{NdPd}_{3} \mathrm{~B}$ & $4 \cdot 187$ \\
\hline $\mathrm{SmPd}_{3}$ & $4 \cdot 110$ & $\mathrm{SmPd}_{3} \mathrm{~B}$ & $4 \cdot 156$ \\
\hline $\mathrm{EuPd}_{3}$ & $4 \cdot 102$ & $\mathrm{EuPd}_{3} \mathrm{~B}$ & $4 \cdot 196$ \\
\hline $\mathrm{GdPd}_{3}$ & 4.087 & $\mathrm{GdPd}_{3} \mathrm{~B}$ & $4 \cdot 146$ \\
\hline $\mathrm{TbPd}_{3}$ & 4.080 & $\mathrm{TbPd}_{3} \mathrm{~B}$ & $4 \cdot 138$ \\
\hline DyPd $_{3}$ & 4.073 & $\mathrm{DyPd}_{3} \mathrm{~B}$ & $4 \cdot 132$ \\
\hline $\mathrm{HoPd}_{3}$ & 4.067 & $\mathrm{HoPd}_{3} \mathrm{~B}$ & $4 \cdot 126$ \\
\hline $\mathrm{ErPd}_{3}$ & 4.061 & $\operatorname{ErPd}_{3} B$ & $4 \cdot 121$ \\
\hline $\mathrm{TmPd}_{3}$ & 4055 & $\operatorname{TmPd}_{3} \mathrm{~B}$ & $4 \cdot 111$ \\
\hline $\mathrm{YbPd}_{3}$ & 4.050 & $\mathrm{YbPd}_{3} \mathrm{~B}$ & $4 \cdot 088$ \\
\hline $\mathrm{LuPd}_{3}$ & $4 \cdot 045$ & $\mathrm{LuPd}_{3} \mathrm{~B}$ & $4 \cdot 090$ \\
\hline
\end{tabular}

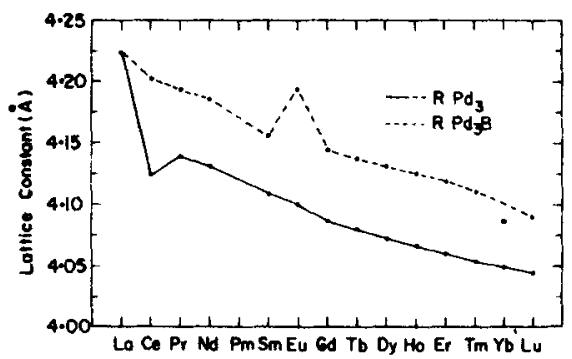

Figure 4. Lattice parameter of $\mathbf{R P d}_{3}$ and $\mathbf{R P d} \mathbf{B}$ ( $\mathbf{R}=$ rare earth) compounds.

\subsection{Magnetic susceptibility measurements}

From figure 4 it is seen that with the exception of EuPd ${ }_{3} B$, the lattice constant of other $\mathrm{RPd}_{3} \mathrm{~B}$ compounds follows the usual lanthanide contraction for trivalent rare earth ions. Susceptibility measurements carried out on some of these compounds, i.e. for $\mathbf{R}=\mathrm{Pr}, \mathrm{Nd}, \mathrm{Sm}, \mathrm{Gd}, \mathrm{Tb}, \mathrm{Dy}, \mathrm{Er}$ and $\mathrm{Tm}$, in the temperature range 77 to $300 \mathrm{~K}$, confirm the trivalency of rare earth ions. For $\mathrm{GdPd}_{3}$ and $\mathrm{GdPd}_{3} \mathrm{~B}$ susceptibility behaviour down to $4.2 \mathrm{~K}$ was recorded for reasons given below. (The addition of boron to $\mathrm{CePd}_{3}$ and $\mathrm{EuPd}_{3}$ results in unusual lattice expansion and changes the magnetic properties of $\mathrm{Ce}$ and Eu ions drastically; results are discussed elsewhere (Dhar et al 1981b; 1982).

With the exception of $\mathrm{SmPd}_{3} \mathrm{~B}$ all other $\mathrm{RPd}_{3} \mathrm{~B}$ compounds, investigated for magnetic behaviour, show a linear $\chi^{-1}$ vs $T$ behaviour (figures 5 and 6). The effective paramagnetic moments were determined by using the formula $\chi(T)=N \mu_{\text {eff }}^{2} /\left[3 k_{B}\left(T-\theta_{p}\right)\right]$. The values of $\mu_{\text {eff }}$ thus obtained are seen to be almost equal to the free ion value for the trivalent state and are listed in table 2 . Small $\theta_{p}$ values suggest that magnetic ordering in $\mathrm{RPd}_{3} \mathrm{~B}$ compounds may occur only at very low temperatures. The nonlinear behaviour of $\chi^{-1}$ vs $T$ observed in $\operatorname{SmPd}_{3} \mathrm{~B}$ (figure 6) is a 


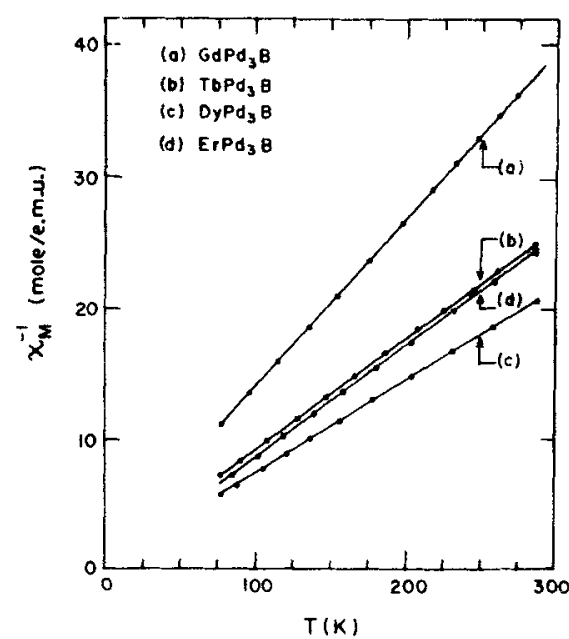

Figure 5. The plot of inverse susceptibility vs temperature of $\mathrm{RPd}_{3} \mathrm{~B}(\mathrm{R}=\mathrm{Gd}, \mathrm{Tb}, \mathrm{Dy}$ and $\mathrm{Er})$ compounds.

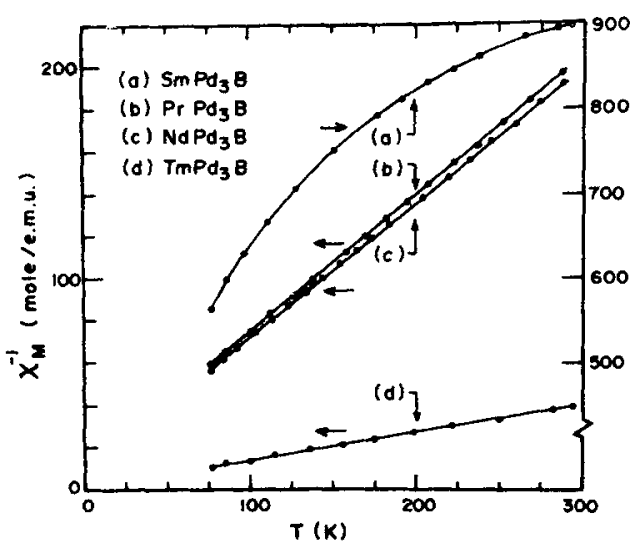

Figure 6. The temperature dependence of inverse susceptibility of $R P_{3} B(R=S m, P r, N d$ and $T m)$ compounds.

Table 2. The effective paramagnetic moment $\mu_{\text {eff }}$ and paramagnetic Curie temperature $\theta_{p}$ in some $R_{P d} B$ and $R R_{3} B(R=$ rare earth) compounds.

\begin{tabular}{lrr}
\hline Compound & $\mu_{\mathrm{eff}}\left(\mu_{B}\right)$ & $\theta_{p}(K)$ \\
\hline $\mathrm{NdPd}_{3} \mathrm{~B}$ & 3.54 & -12.0 \\
$\operatorname{PrPd}_{3} \mathrm{~B}$ & 3.54 & -13.0 \\
$\mathrm{TbPd}_{3} \mathrm{~B}$ & 9.76 & -11.0 \\
$\mathrm{DyPd}_{3} \mathrm{~B}$ & 10.73 & -7.8 \\
$\mathrm{ErPd}_{3} \mathrm{~B}$ & 9.74 & -5.0 \\
$\mathrm{TmPd}_{3} \mathrm{~B}$ & 7.76 & -4.0 \\
$\mathrm{NdRh}_{3} \mathrm{~B}$ & 3.75 & -7.0 \\
$\operatorname{PrRh}_{3} \mathrm{~B}$ & 3.80 & -18.0 \\
$\mathrm{TbRh}_{3} \mathrm{~B}$ & 9.77 & 5.0 \\
$\mathrm{DyRh}_{3} \mathrm{~B}$ & 10.69 & 4.0 \\
$\mathrm{ErRh}_{3} \mathrm{~B}$ & 9.64 & 5.0 \\
\hline-
\end{tabular}

consequence of the temperature independent contribution arising due to narrow multiplet separation of $\mathrm{Sm}^{3+}$ ion.

Introduction of non magnetic boron in the $\mathrm{RPd}_{3}$ compounds, besides expanding the lattice, will also change the electron concentration in the conduction band. Since RKKY interaction responsible for magnetic ordering in rare earth compounds is electron concentration-dependent, any change in the latter would be expected to modify the exchange interaction between the rare earth ions. Examples exist in literature where such effects have been found. For instance while the moment carrying trivalent rare earths $\mathrm{RAl}_{2}$ order ferromagnetically (except $\mathrm{CeAl}_{2}$ ), the compound $\mathrm{EuAl}_{2}$ in which $\mathrm{Eu}$ is divalent and, therefore, contributes one electron less to the conduction band, is 
antiferromagnetic (Wallace 1973). In the pseudobinary $\mathrm{Eu}_{x} \mathrm{La}_{1-x} \mathrm{Al}_{2}$ a reversal in the magnetic coupling from antiferromagnetic to ferromagnetic with increasing $x$, which alters the electron concentration, is observed (Mader and Wallace 1968). Similarly while GdAg and GdIn order antiferromagnetically with Neel temperatures of $145 \mathrm{~K}$ and $28 \mathrm{~K}$ respectively, the pseudobinary solutions $\mathrm{GdAg}_{x} \operatorname{In}_{1-x}$ exhibit ferromagnetism for $0.8<x<0.3$ with Curie temperature increasing with increasing $x$ (Sekizawa and Yasukochi 1966). The dependence of exchange interaction on the electron concentration can be understood from the formula

$$
\theta_{p}=-\frac{3 \pi Z^{2}|J(0)|^{2}}{k_{B} E_{F}} S(S+1) \sum_{i} F\left(2 k_{r} r_{i}\right),
$$

where $\theta_{p}$ is the paramagnetic Curie temperature, $Z$ is the average number of conduction electrons per atom, $2 J(0)$ is the exchange interaction constant between the rare earth spin $S$ and conduction electron spin $s, E_{F}$ is the Fermi energy and $F(x)=(x \cos x-\sin x) / x^{4}$. In the molecular field approximation $\theta_{p}$ is identical with the magnetic ordering temperature. The sign of $F(x)$ determines the kind of magnetic order that develops: antiferromagnetic if sum is positive and ferromagnetic if the sum is negative. With the increase in electron concentration, i.e. an increase in $Z$ and hence in $k_{F}$ (assuming crystal structure remains the same) $x$ increases and $F(x)$ shows an oscillatory behaviour from positive to negative values.

In order to see if the addition of boron is accompanied with any electron concentration effects, magnetic behaviour of $\mathrm{GdPd}_{3}$ and $\mathrm{GdPd}_{3} \mathrm{~B}$ was studied down to liq.He temperature. The compound $\mathrm{GdPd}_{3}$ was chosen because it is the only compound in the $\mathrm{RPd}_{3}$ series that orders magnetically above $4 \cdot 2 \mathrm{~K}$ (Gardner et al 1972). Figure 7 shows the magnetisation as a function of temperature in $\mathrm{GdPd}_{3}$ and $\mathrm{GdPd}_{3} \mathrm{~B}$. In an external field of $4 \mathrm{kOe}$ magnetisation of $\mathrm{GdPd}_{3}$ shows a sharp singularity at $\sim 6 \mathrm{~K}$ which corresponds to an antiferromagnetic ordering in this compound. No such feature is observed in $\mathrm{GdPd}_{3} \mathrm{~B}$ which continues to remain paramagnetic down to $4.2 \mathrm{~K}$. These observations show that the addition of boron modifies the exchange interaction

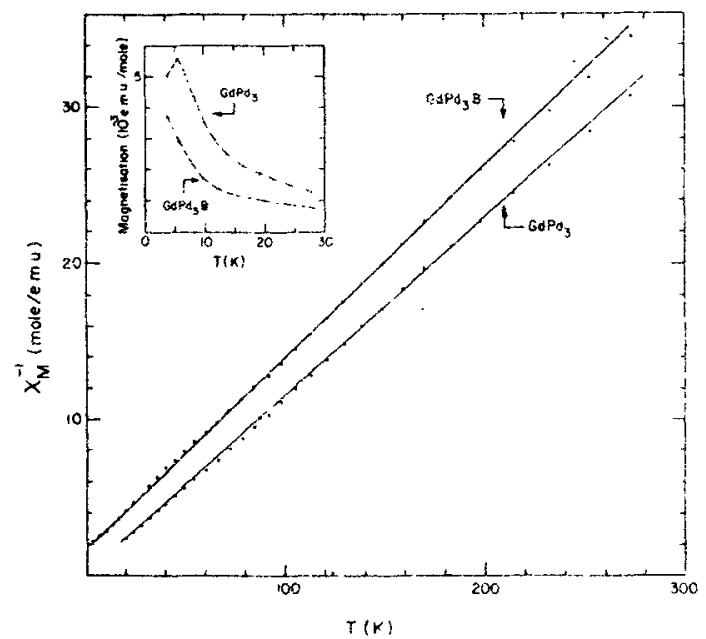

Figure 7. The inverse susceptibility of $\mathrm{GdPd}_{3}$ and $\mathrm{GdPd}_{3} \mathrm{~B}$ as a function of temperature. The inset shows the temperature dependence of magnetization (in a field of $4 \mathrm{kOe}$ ). 
between the rare earth ions either due to lattice expansion, or by changing electron concentration or a combination of both. Extension of magnetisation below $4.2 \mathrm{~K}$ may, perhaps, show evidence of magnetic ordering in $\mathrm{GdPd}_{3} \mathrm{~B}$.

\section{3. $R R h_{3} B$ compounds}

\subsection{Synthesis and structure}

In the beginning of this paper we mentioned that alloys of the formula $\mathrm{CeRh}_{3} \mathrm{~B}_{x}$ could be formed. This observation together with the fact that alloys of the composition $\mathbf{R P d}_{3} \mathbf{B}_{x}$ exist for all $\mathrm{R}$ prompted us to investigate whether single phase $R \mathbf{R h}_{3} \mathbf{B}_{x}$ alloys could be synthesised. It was found that $R \mathrm{Rh}_{3} \mathrm{~B}$ compounds, do indeed, form for all rare earths $R$. The $x$-ray diffraction patterns of $R R_{3} B$ compounds (figure 8) are similar to those of $R \mathrm{Rd}_{3} \mathrm{~B}$ compounds. Therefore, presumably $R \mathrm{Rh}_{3} \mathrm{~B}$ compounds also crystallise in the anti-perovskite or filled $\mathrm{AuCu}_{3}$ type structure. It may be noted that while the parent $R \mathbf{P d}_{3}$ compounds exist for all $R$, such is not the case with $R R h_{3}$ compounds. The latter form only for $\mathrm{R}=\mathrm{Ce}\left(\mathrm{AuCu}_{3}\right.$ type structure) and $\mathrm{La}, \mathrm{Sm}, \mathrm{Nd}$ and $\mathrm{Gd}$ (hexagonal $\mathrm{CeNi}_{3}$ type structure) (Iandelli and Palenzona 1979). The non-metallic element boron, therefore, has the effect of stabilising the cubic $\mathrm{RRh}_{3} \mathrm{~B}$ phase. In fact, while our experimental investigations were on, we came across instances in the literature where complex metallic phases are stabilised by atoms like C, B, N, Si etc (Stadelmaier 1979). The alloys of composition $\mathrm{RRh}_{3} \mathrm{C}_{x}(x \sim 1)$ for all $\mathrm{R}$ also exist in the perovskite structure (Holleck 1971), thus strengthening our conclusions, reached independently by us, about the crystal structure of $R R h_{3} B$ compounds.

In contrast to $\mathrm{RPd}_{3} \mathrm{~B}_{x}$ alloys where $0<x \leqslant 1$, it appears that $\mathrm{RRh}_{3} \mathrm{~B}_{x}$ compounds do not form below a certain minimum concentration of boron $\left(C e \mathrm{Rh}_{3} \mathrm{~B}_{x}\right.$ being an exception). Thus for $x=0.5$ no perovskite formation was observed for $\mathrm{R}=\mathrm{La}, \mathrm{Nd}$ and Er. Though, this was not tested for other rare earths we believe similar considerations should hold. We have not determined the minimum value of $x$ for which $R R h_{3} B_{x}$ alloys can be stabilised.

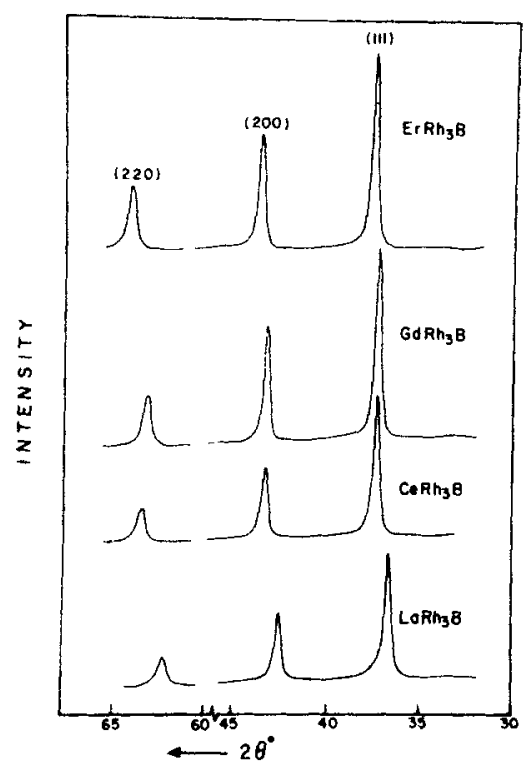

Figure 8. The $x$-ray diffraction patterns of some $\mathrm{RRh}_{3} \mathrm{~B}$ ( $\mathrm{R}=\mathrm{La}, \mathrm{Ce}, \mathrm{Gd}$ and $\mathrm{Er}$ ) compounds. These patterns can be indexed on the basis of a cubic unit cell. 


\subsection{Magnetic susceptibility and ${ }^{151} E u$ Mössbauer measurements}

The lattice constants of $R R h_{3} B$ compounds are plotted in figure 9. With the exception of $\mathrm{CeRh}_{3} \mathrm{~B}$, the usual lanthanide contraction with no anomaly in the lattice parameter is observed. This points out the trivalent character of rare earth ions in these compounds. This has been verified from susceptibility measurements carried out on some of these compounds in the temperature range 300 to $77 \mathrm{~K}$. The $\chi^{-1}$ vs $T$ plots are shown in figures 10 and 11. For $\mathrm{SmRh}_{3} \mathrm{~B} \chi^{-1}$ vs $T$ is non-linear for reasons similar to those given for $\mathrm{SmPd}_{3} B$. The effective paramagnetic moments (very close to free ion value) and paramagnetic Curie temperatures are listed in table 2 .

In case of $E u R h_{3} B$, the trivalency of Eu has been confirmed from ${ }^{151} \mathrm{Eu}$ Mössbauer spectroscopy. A single Mössbauer absorption with isomer shift of $2.4 \pm 0.1 \mathrm{~mm} / \mathrm{sec}$, characteristic of trivalent Eu in metallic environments, is observed at $300 \mathrm{~K}$ (figure 12). There is no change in isomer shift on lowering the temperature to $88 \mathrm{~K}$.

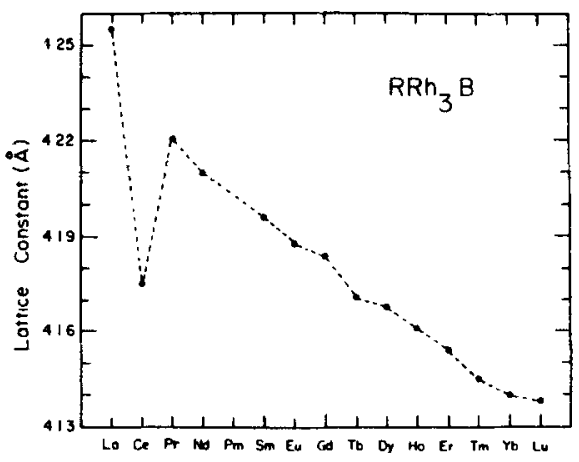

Figure 9. The lattice parameter of $R \mathbf{R h}_{3} \mathbf{B}$ ( $R=$ rare earth) compounds.

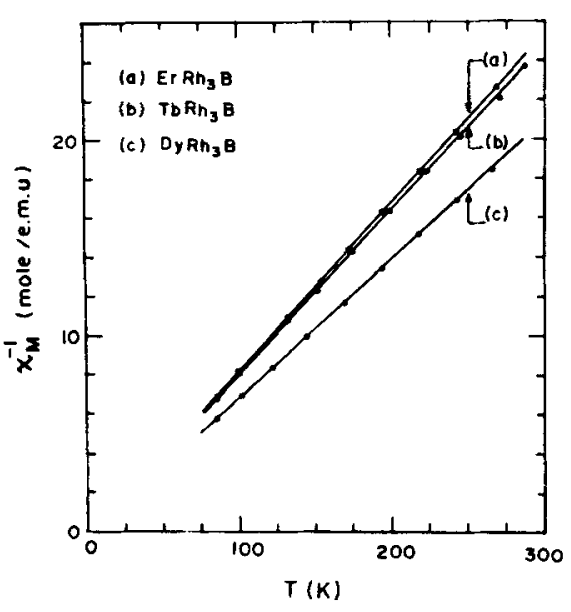

Figure 10. Inverse susceptibility as a function of temperature in $R_{R h} B(R=E r, T b$ and $D y)$ compounds.

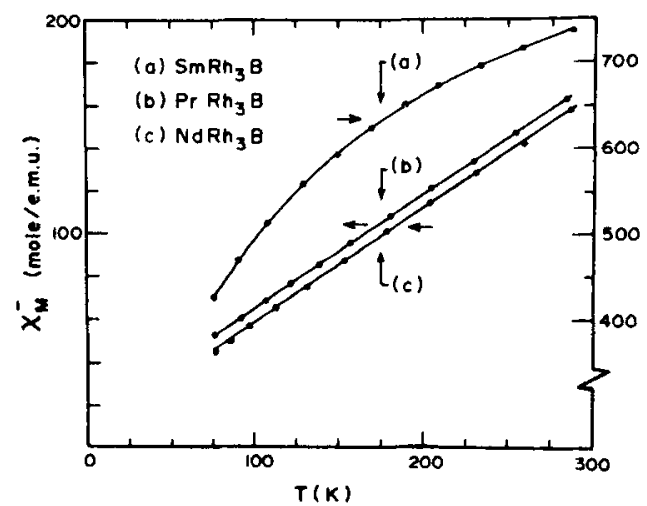

Figure 11. Inverse susceptibility $v$ s temperature of $R_{R} h_{3} B(R=S m, \operatorname{Pr}$ and Nd) compounds. 


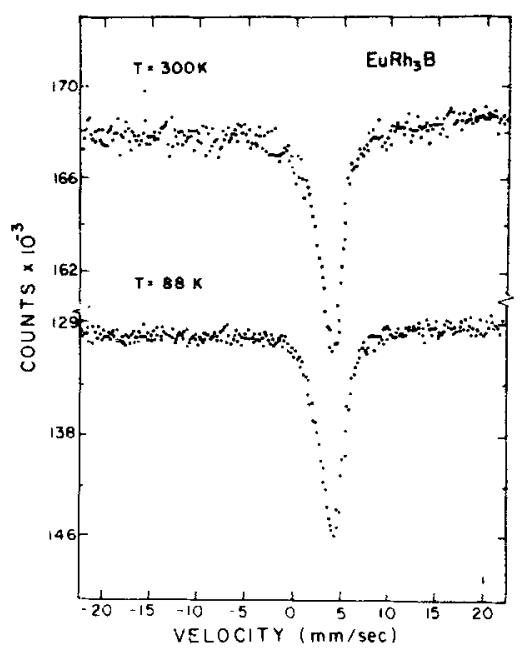

Figure 12. ${ }^{151} \mathrm{Eu}$ Mössbauer spectrum of $\mathrm{EuRh}_{3} \mathrm{~B}$ at 300 and $88 \mathrm{~K}$.

\section{Silicon addition to $\operatorname{RPd}_{3}$ compounds}

From the structural considerations of the $\mathrm{RPd}_{3}$ unit cell mentioned above, we concluded that the small boron atoms in the $\operatorname{RPd}_{3} \mathbf{B}_{x}$ alloys occupy the interstitial vacant body-centred position. It was, therefore, natural to investigate whether other non-metallic elements could be introduced in the $\mathrm{RPd}_{3}$ lattice. In this direction we tried the synthesis of $\mathrm{RPd}_{3} \mathrm{Si}_{x}$ alloys for $\mathrm{R}=\mathrm{La}, \mathrm{Ce}$ and $\mathrm{Eu}$ only. It was found that like boron, silicon can also be alloyed with $\mathrm{RPd}_{3}$ compounds. However, the atomic percentage of silicon that can be dissolved into $\mathbf{R P d}_{3}$ lattice is far less than that of boron. Only up to $x \sim 0.3$, single phase cubic alloys were obtained for $\operatorname{RPd}_{3} \mathrm{Si}_{x}$ composition. This may be related to the relatively larger atomic volume of silicon compared to that of boron. From volume filling considerations alone it may not be possible to accommodate as many number of silicon atoms as boron. The addition of silicon, like boron, to parent $\mathrm{RPd}_{3}$ compound leads to lattice expansion. Results of magnetic susceptibility and ${ }^{151} \mathrm{Eu}$ Mössbauer spectroscopy on $\mathrm{CePd}_{3} \mathrm{Si}_{x}$ and $\mathrm{EuPd}_{3} \mathrm{Si}_{x}$, where a valence change of $\mathrm{Ce}$ and Eu ions is observed on alloying with $\mathrm{Si}$, are presented elsewhere (Malik et al 1982; Dhar et al 1983).

Carbon addition to $\mathrm{RPd}_{3}$ compounds was also tried and it was found that carbon cannot be alloyed with $\mathrm{RPd}_{3}$ compounds.

\section{References}

Dhar S K, Malik S K and Vijayaraghavan R 1981a Mater. Res. Bull. 161557

Dhar S K, Malik S K and Vijayaraghavan R 1981b Phys. Rev. B24 6182

Dhar S K, Malik S K, Rambabu D and Vijayaraghavan R 1982 J. Appl. Phys. 538077

Dhar S K, Nagarajan R, Malik S K, Rambabu D and Vijayaraghavan R 1983 J. Magn. \& Magn. Mater. 393 31-34

Gardner W E, Penfold J, Smith T F and Harris I R 1972 J. Phys. F2 133

Gschneidner Jr K A, MaMasters O D and Ostenson J E 1978 J. Less-Common Metals 62167

Harris I R and Raynor G V 1965 J. Less Common-Metals 9263

Harris I R and Norman M 1967 J. Less Common-Metals 13629 
Holleck H 1971 in Rare earths and actinides (ed) E W Lee (London: Creative Offset Ltd.) p. 109 Iandelli A and Palenzona A 1979 in Handbook on the physics and chemistry of rare earths (eds) K. A Gschneidner Jr and L Erying Ch. 13 (Amsterdam: North Holland)

Mader K H and Wallace W E 1968 J. Chem. Phys. 491521

Malik S K, Vijayaraghavan R, Boltich E B, Craig R S, Wallace W E and Dhar S K 1982 Solid State Commun. 43243

Malik S K, Vijayaraghavan R, Wallace W E and Dhar S K 1983 J. Magn. \& Magn. Mater. 37303

Matthias B T, Corenzwit E, Vandenberg J M and Barz H E 1977 Proc. Nat. Acad. Sci. USA 741334

Pearson W B 1967 Handbook of lattice spacings and structure of metals (New York: Pergamon) Vol. 2, p. 56, 57

Sekizawa K and Yasukochi K 1966 J. Phys. Soc. Jpn 21684

Stadelmaier H H 1969 in Developments in the structural chemistry of alloy phases (ed.) B C Giessen (New York: Plenum)

Vandenberg J M and Matthias B T 1977 Proc. Natl. Acad. Sci. USA 741336 